NOTICE: this is the author's version of a work that was accepted for publication in Signal Processing. Changes resulting from the publishing process, such as peer review, editing, corrections, structural formatting, and other quality control mechanisms may not be reflected in this document. Changes may have been made to this work since it was submitted for publication. A definitive version was subsequently published in Signal Processing, Vol. 93, No. 7 (2013). DOI: 10.1016/j.sigpro.2013.01.015 


\title{
A Direct Optimization Method for Low Group Delay FIR Filter Design
}

\author{
Changzhi $\mathrm{Wu}$ and David Gao \\ School of Science, Information, Technology and Engineering, \\ University of Ballarat, Australia \\ Kok Lay Teo \\ Department of Mathematics and Statistics, Curtin University, Australia
}

\begin{abstract}
This paper studies the design of FIR filter with low group delay, where the desired phase response is not being approximated. It is formulated as a constrained optimization problem, which is then solved globally. Numerical experiments show that our design method can produce a filter with smaller group delay than that obtained by the existing convex optimization method used in conjunction with a minimum phase spectral factorization method under the same design criteria. Furthermore, our formulation offers us the flexibility for the trade-off between the group delay and the magnitude response directly. It also allows the feasibility of imposing constraints on the group delay.
\end{abstract}

Keywords: Low group delay, duality, semi-definite programming 


\section{Introduction}

For filters with the same filter lengths, it is well known that nonlinear phase FIR filters can have smaller group delay and achieve better frequency selectivity than linear phase FIR filters if they are designed properly. Thus, nonlinear phase FIR filters have a wide range of applications in communications $[1,2]$. A representative example is in telecommunications where long delays are known to cause problems of echo and singing on voice lines [3].

There are many methods available in the literature for nonlinear phase FIR filter design [1-13]. For the design of FIR filter with desired phase response, it can be formulated either as a convex quadratic optimization problem with convex quadratic constraints [4] or as a nonconvex optimization problem [2], depending on whether or not the constraints on the group delay are imposed directly. For the case without constraints being imposed on its group delay, the formulated quadratic convex optimization problems in [4] are easy to solve by existing software packages. However, if the constraints on the group delay are imposed directly, the formulated optimization problem in [2] is highly complicated and only local optimal solutions can be ensured. To overcome this difficulty, the non-convex constraints on group delay are relaxed to convex constraints such that the relaxed optimization problem can be solved by the semi-definite programming (SDP) [5]. Although a good solution can be obtained in some cases, there is no guarantee that it will do 
the same in general.

For the design of FIR filter without taking into consideration of its group delay, it can be formulated as a semi-definite quadratic optimization problem with linear constraints (see [6] and [7]). By solving this convex programming problem, the autocorrelation of the designed filter is obtained. Then, a spectral factorization procedure is applied to construct the original filter. If a low group delay is required, then a minimum phase extraction method is applied. Traditionally, the design of such a filter is referred to as the minimum phase FIR filter design. There are many methods, which are available for the minimum phase filter design [3-8]. The methods in [8] and [9] are mainly based on Herrmann's design procedure [10] in which the frequency response of odd-length equiripple linear-phase prototype filter is shifted up by onehalf of its stopband's peak-to-peak ripple to acquire the second-order zeros on the unit circle of the $z$-plane. In [3], a design procedure is proposed to design minimum-phase filter based on root moments while keeping the same magnitude response. However, it requires to start from a linear-phase filter. In [11], a design procedure from a mixed-phase filter is proposed to fill the drawback in [3]. All the aforementioned methods for the minimum phase filter design are required to have a given FIR filter. On this basis, its minimum phase part are being extracted. The smallest group delay of the minimum phase filter is obtained from the family of filters with the same magnitude 
response. For a general constrained filter design, the smallest group delay cannot be obtained through the design of a minimum phase filter.

In this paper, we study the design of a low group delay FIR filter without imposing constraints on its phase response. Unlike the conventional minimum phase FIR filter design [3-8], we formulate it as an optimization problem directly and solve it globally. Numerical experiments show that our design method can produce a filter with smaller group delay than those obtained by the existing convex optimization method used in conjunction with a minimum phase extraction method under the same design criteria. Our formulation offers us the flexibility for the trade-off between the group delay and the magnitude response. Furthermore, it allows the feasibility of imposing constraints on the group delay. To achieve this task, we first derive a new simple criterion for group delay. Based on this newly derived criterion, the design of a low group delay nonlinear phase FIR filter is formulated as a fourth order polynomial optimization problem with quadratic constraints. However, the solution of this problem is difficult to obtain, as solving the $p$ norm FIR filter design problem is a challenge. The main contribution of this paper is a novel and simple computational scheme to solve this difficult optimization problem based on the canonical duality developed in [15]. Since the objective function and the constraints for our formulated problem are either quadratic or the composite of two quadratic functions, the solution of the for- 
mulated optimization problem may be non-unique, causing difficulty in its convex reformulation [16]. To tackle this issue, we introduce a small linear perturbation term to break its symmetry. Under the canonical duality theory [15], the global solution of this linearly perturbed optimization problem is obtained via solving a simple SDP problem. Since the perturbation term is very small, the global solution of the perturbed problem can be viewed as an approximate global solution of the original problem. To achieve better performance, a gradient-based optimization method can be applied to refine the current approximate global solution. Numerical experiments are presented to compare the results obtained by our method and those obtained by exiting methods. From the comparison, it is clearly observed that our method offers flexibility on the trade-off between group delay and the magnitude response. This feature is not shared by the existing methods. Furthermore, our method gives rise to a smaller group delay than those obtained by existing methods under the same design criteria.

\section{Problem Formulation}

Consider an FIR digital filter of order $K$ with transfer function $H(z)$ given by

$$
H(z)=\sum_{k=0}^{K} h_{k} z^{-k}
$$


where $h_{k}, k=0,1, \ldots, K$, are real filter coefficients. The frequency response $H(\omega)$ can be expressed as

$$
H(\omega)=|H(\omega)| e^{j \phi(\omega)},
$$

where $|H(\omega)|$ denotes the magnitude of $H(\omega)$, while $\phi(\omega)$ is the phase response which is related to the group delay $\tau(\omega)$ as shown by

$$
\tau(\omega)=-\frac{d \phi(\omega)}{d \omega}
$$

Let $\left|H_{d}(\omega)\right|$ be the desired magnitude response given by

$$
\left|H_{d}(\omega)\right|= \begin{cases}1, & \text { if } \omega \in B_{p} \\ 0, & \text { if } \omega \in B_{s}\end{cases}
$$

where $B_{p}$ is the passband and $B_{s}$ is the stopband. Taking derivative on both sides of equation (2), we obtain

$$
\frac{d H(\omega)}{d \omega}=-j \tau(\omega) H(\omega)+\frac{d|H(\omega)|}{d \omega} e^{j \phi(\omega)}
$$

In light of (1), we have

$$
\sum_{k=0}^{K} k h_{k} e^{-j k \omega}=j \frac{d H(\omega)}{d \omega}
$$

By the Parseval equality, it gives

$$
\begin{aligned}
& \sum_{k=0}^{K} k h_{k}^{2}=\frac{1}{2 \pi} \int_{-\pi}^{\pi}\left(j \frac{d H(\omega)}{d \omega}\right) H^{*}(\omega) d \omega \\
= & \frac{1}{2 \pi} \int_{-\pi}^{\pi} \tau(\omega)|H(\omega)|^{2} d \omega+j \frac{1}{2 \pi} \int_{-\pi}^{\pi} \frac{d|H(\omega)|}{d \omega}|H(\omega)| d \omega
\end{aligned}
$$


where $H^{*}(\omega)$ is the conjugate of $H(\omega)$. Since $|H(\omega)|$ is an even function, it follows that

$$
\int_{-\pi}^{\pi} \frac{d|H(\omega)|}{d \omega}|H(\omega)| d \omega=0
$$

Thus,

$$
\sum_{k=0}^{K} k h_{k}^{2}=\frac{1}{2 \pi} \int_{-\pi}^{\pi} \tau(\omega)|H(\omega)|^{2} d \omega \approx \frac{1}{2 \pi} \int_{B_{p} \cup B_{t}} \tau(\omega)|H(\omega)|^{2} d \omega,
$$

where $B_{t}$ is the transition band. In (8), it is shown that $\sum k h_{k}^{2}$ is a good measurement of the group delay in the passband and the transition band. Our extensive numerical experiments show that minimization of $\sum k h_{k}^{2}$ can achieve a desired low group delay filter in the passband. This formula has been used in [17] to design narrow band lowpass filters with low group delay. Let $\boldsymbol{h}=\left[h_{0}, h_{1}, \cdots, h_{K}\right]^{T}$ and $D=\operatorname{diag}(0,1, \cdots, K)$. Now we pose the design of a low group delay filter with the prescribed magnitude responses given by (4) as the following optimization problem

$$
\begin{aligned}
& \min _{\boldsymbol{h}} P(\boldsymbol{h})=\alpha \boldsymbol{h}^{T} D \boldsymbol{h}+(1-\alpha) \int_{B_{p}}\left(|H(\omega)|^{2}-1\right)^{2} d \omega \\
& \text { subject to }|H(\omega)|^{2} \leq \varepsilon, \forall \omega \in B_{s}
\end{aligned}
$$

where $\alpha$ is a weighting factor and $\varepsilon$ is a ripple specification in the stopband. Let this problem be referred to as Problem $(\mathrm{P})$. In the absence of the first quadratic term in $P(\boldsymbol{h})$, Problem $(\mathrm{P})$ can be reformulated as a new quadratic optimization problem with linear constraints expressed in terms of the autocorrelation of the designed filter $H(z)([6],[7])$. By solving this reformulated 
quadratic optimization problem with linear constraints together with spectral factorization, the original filter coefficients are obtained. During the computation process, only linear matrix inequality (LMI) and spectral factorization are involved. Thus, the method proposed in [6] and [7] is effective. However, due to the presence of the first quadratic term in (9), this method is not applicable to Problem $(\mathrm{P})$. In the following sections, we will develop an efficient method to solve Problem (P).

\section{Solution Method}

During the computational process, the one-dimensional integral that appeared in (9) is evaluated by using Gaussian quadrature with $M$ nodes, i.e.,

$\int_{B_{p}}\left(|H(\omega)|^{2}-1\right)^{2} d \omega=\sum_{m=1}^{M} \nu_{m}\left(\left|H\left(\omega_{m}^{p}\right)\right|^{2}-1\right)^{2}=\sum_{m=1}^{M} \nu_{m}\left(\boldsymbol{h}^{T} \Psi\left(\omega_{m}^{p}\right) \boldsymbol{h}-1\right)^{2}$,

where $\nu_{m}, m=1, \cdots, M$, are weights, $\omega_{m}^{p}, m=1, \cdots, M$, are the Gaussian

quadrature points, $\Psi(\omega)=C(\omega) C^{T}(\omega)+S(\omega) S^{T}(\omega), C(\omega)=[1, \cos (\omega), \cdots, \cos (K \omega)]^{T}$ and $S(\omega)=[0, \sin (\omega), \cdots, \sin (K \omega)]^{T}$. The functional inequality constraint (10) is discretized into finite number of constraints given below:

$$
\left|H\left(\omega_{n}^{s}\right)\right|^{2}=\boldsymbol{h}^{T} \Psi\left(\omega_{n}^{s}\right) \boldsymbol{h} \leq \varepsilon, \quad n=1, \cdots, N
$$

where $\omega_{n}^{s} \in B_{s}, \quad n=1, \cdots, N$, and $N$ is the number of the discretization points. Now Problem (P) is approximated by the following optimization 
problem.

$$
\begin{aligned}
& \min _{\boldsymbol{h}} \widetilde{P}(\boldsymbol{h})=\alpha \boldsymbol{h}^{T} D \boldsymbol{h}+(1-\alpha) \sum_{m=1}^{M} \nu_{m}\left(\boldsymbol{h}^{T} \Psi\left(\omega_{m}^{p}\right) \boldsymbol{h}-1\right)^{2} \\
& \text { subject to } \quad \boldsymbol{h}^{T} \Psi\left(\omega_{n}^{s}\right) \boldsymbol{h} \leq \varepsilon, n=1, \cdots, N .
\end{aligned}
$$

Let this problem be referred to as Problem $\left(P_{I}\right)$. Since (13) and (14) are all symmetric with respect to $\boldsymbol{h}$, the solution of Problem $\left(P_{I}\right)$ may not be unique which could lead to some difficulties in its convex reformulation. To break the symmetry, we introduce a linear small perturbation to (13) as it was suggested in [18]. Under this linear perturbation, (13) becomes

$$
\tilde{P}_{\boldsymbol{g}}(\boldsymbol{h})=\alpha \boldsymbol{h}^{T} D \boldsymbol{h}+(1-\alpha) \sum_{m=1}^{M} \nu_{m}\left(\boldsymbol{h}^{T} \Psi\left(\omega_{m}^{p}\right) \boldsymbol{h}-1\right)^{2}-\boldsymbol{g}^{T} \boldsymbol{h},
$$

where $\boldsymbol{g}$ is a small perturbation vector. Replacing (13) by (15) in Problem $\left(P_{I}\right)$, we obtain a perturbed optimization problem which is referred to as Problem $\left(P_{I g}\right)$. If $\boldsymbol{g}$ is small enough, then the global solution of Problem $\left(P_{I g}\right)$ can be viewed as an approximate global solution of Problem $\left(P_{I}\right)$. Thus, it is of crucial importance if Problem $\left(P_{I g}\right)$ can be solved efficiently. In the following, we introduce the canonical duality developed in [15] to solve Problem $\left(P_{I g}\right)$.

The Lagrangian function for Problem $\left(P_{I g}\right)$ is defined as

$$
\begin{aligned}
& L(\boldsymbol{h}, \boldsymbol{\lambda})=\alpha \boldsymbol{h}^{T} D \boldsymbol{h}+(1-\alpha) \sum_{m=1}^{M} \nu_{m}\left(\boldsymbol{h}^{T} \Psi\left(\omega_{m}^{p}\right) \boldsymbol{h}-1\right)^{2}-\boldsymbol{g}^{T} \boldsymbol{h} \\
& +\sum_{n=1}^{N} \lambda_{n}\left(\boldsymbol{h}^{T} \Psi\left(\omega_{n}^{s}\right) \boldsymbol{h}-\varepsilon\right)
\end{aligned}
$$


where $\boldsymbol{\lambda} \geq 0 . \boldsymbol{h}^{*}$ is called a KKT point of Problem $\left(P_{I g}\right)$ if there exists a $\boldsymbol{\lambda}^{*}$ such that

$$
\left\{\begin{array}{l}
\nabla_{\boldsymbol{h}} L\left(\boldsymbol{h}^{*}, \boldsymbol{\lambda}^{*}\right)=0, \\
\lambda_{n}^{*}\left(\left(\boldsymbol{h}^{*}\right)^{T} \Psi\left(\omega_{n}^{s}\right) \boldsymbol{h}^{*}-\varepsilon\right)=0, \quad n=1, \cdots, N, \\
\boldsymbol{\lambda}^{*}=\left[\lambda_{1}^{*}, \cdots, \lambda_{N}^{*}\right]^{T} \geq 0 .
\end{array}\right.
$$

Let $\xi_{m}=\boldsymbol{h}^{T} \Psi\left(\omega_{m}^{p}\right) \boldsymbol{h}-1, m=1, \cdots, M$, and $V(\boldsymbol{\xi})=\boldsymbol{\xi}^{T} B \boldsymbol{\xi}$, where $\boldsymbol{\xi}=\left[\xi_{1}, \cdots, \xi_{M}\right]^{T}$ and $B=(1-\alpha) \operatorname{diag}\left\{\nu_{1}, \cdots, \nu_{M}\right\}$. Clearly, $V(\boldsymbol{\xi})$ is a strictly convex function. Thus, (16) can be rewritten as

$$
\bar{L}(\boldsymbol{h}, \boldsymbol{\xi}, \boldsymbol{\lambda})=\alpha \boldsymbol{h}^{T} D \boldsymbol{h}+V(\boldsymbol{\xi})+\sum_{n=1}^{N} \lambda_{n}\left(\boldsymbol{h}^{T} \Psi\left(\omega_{n}^{s}\right) \boldsymbol{h}-\varepsilon\right)-\boldsymbol{g}^{T} \boldsymbol{h} .
$$

Let $\boldsymbol{\varsigma}=\nabla V(\boldsymbol{\xi})=2 B \boldsymbol{\xi}$. The Fenchel conjugate of $V(\boldsymbol{\xi})$ is defined [19] as

$$
V^{*}(\boldsymbol{\varsigma})=\boldsymbol{\varsigma}^{T} \boldsymbol{\xi}-V(\boldsymbol{\xi})=\frac{1}{4} \boldsymbol{\varsigma}^{T} B^{-1} \boldsymbol{\varsigma}
$$

Replacing $V(\boldsymbol{\xi})$ by $\boldsymbol{\varsigma}^{T} \boldsymbol{\xi}-V^{*}(\boldsymbol{\varsigma})$ in (18), the following total complementary function [15] is obtained.

$\Xi(\boldsymbol{h}, \boldsymbol{\varsigma}, \boldsymbol{\lambda})=\sum_{m=1}^{M} \varsigma_{m}\left(\boldsymbol{h}^{T} \Psi\left(\omega_{m}^{p}\right) \boldsymbol{h}-1\right)-\frac{1}{4} \varsigma^{T} B^{-1} \boldsymbol{\varsigma}+\sum_{n=1}^{N} \lambda_{n}\left(\boldsymbol{h}^{T} \Psi\left(\omega_{n}^{s}\right) \boldsymbol{h}-\varepsilon\right)+\alpha \boldsymbol{h}^{T} D \boldsymbol{h}-\boldsymbol{g}^{T} \boldsymbol{h}$.

Taking $\nabla_{\boldsymbol{h}} \Xi(\boldsymbol{h}, \boldsymbol{\varsigma}, \boldsymbol{\lambda})=0$, we have

$$
2 G(\boldsymbol{\varsigma}, \boldsymbol{\lambda}) \boldsymbol{h}=\boldsymbol{g},
$$

where

$$
G(\boldsymbol{\varsigma}, \boldsymbol{\lambda})=\alpha D+\sum_{m=1}^{M} \varsigma_{m} \Psi\left(\omega_{m}^{p}\right)+\sum_{n=1}^{N} \lambda_{n} \Psi\left(\omega_{n}^{s}\right)
$$


If $G(\boldsymbol{\varsigma}, \boldsymbol{\lambda})$ is invertible, then $\boldsymbol{h}=\frac{1}{2} G^{-1}(\boldsymbol{\varsigma}, \boldsymbol{\lambda}) \boldsymbol{g}$. Substituting this $\boldsymbol{h}$ into (19), we obtain the canonical dual function

$$
P_{I g}^{d}(\boldsymbol{\varsigma}, \boldsymbol{\lambda})=-\frac{1}{4} \boldsymbol{g}^{T} G^{-1}(\boldsymbol{\varsigma}, \boldsymbol{\lambda}) \boldsymbol{g}-\frac{1}{4} \boldsymbol{\varsigma}^{T} B^{-1} \boldsymbol{\varsigma}-\sum_{m=1}^{M} \varsigma_{m}-\varepsilon \sum_{n=1}^{N} \lambda_{n}
$$

The canonical dual problem $\left(P_{I g}^{d}\right)$ is defined as follows:

$$
\max _{\boldsymbol{\varsigma}, \boldsymbol{\lambda}}\left\{P_{I g}^{d}(\boldsymbol{\varsigma}, \boldsymbol{\lambda}) \mid \boldsymbol{\lambda} \geq 0\right\}
$$

The Lagrangian function $\tilde{L}(\boldsymbol{\varsigma}, \boldsymbol{\lambda}, \boldsymbol{\sigma})$ of Problem $\left(P_{I g}^{d}\right)$ is defined as

$$
\tilde{L}(\boldsymbol{\varsigma}, \boldsymbol{\lambda}, \boldsymbol{\sigma})=P_{I g}^{d}(\boldsymbol{\varsigma}, \boldsymbol{\lambda})+\boldsymbol{\sigma}^{T} \boldsymbol{\lambda}
$$

where $\boldsymbol{\sigma} \geq 0 .\left(\boldsymbol{\varsigma}^{*}, \boldsymbol{\lambda}^{*}\right)$ is called a KKT point of Problem $\left(P_{I g}^{d}\right)$ if there exists a $\boldsymbol{\sigma}^{*}$ such that

$$
\left\{\begin{array}{l}
\nabla_{\boldsymbol{\varsigma}} \tilde{L}\left(\boldsymbol{\varsigma}^{*}, \boldsymbol{\lambda}^{*}, \boldsymbol{\sigma}^{*}\right)=0, \nabla_{\boldsymbol{\lambda}} \tilde{L}\left(\boldsymbol{\varsigma}^{*}, \boldsymbol{\lambda}^{*}, \boldsymbol{\sigma}^{*}\right)=0 \\
\left(\boldsymbol{\sigma}^{*}\right)^{T} \boldsymbol{\lambda}^{*}=0 \\
\boldsymbol{\sigma}^{*}=\left[\sigma_{1}^{*}, \cdots, \sigma_{N}^{*}\right]^{T} \geq 0, \boldsymbol{\lambda}^{*}=\left[\lambda_{1}^{*}, \cdots, \lambda_{N}^{*}\right]^{T} \geq 0 .
\end{array}\right.
$$

For Problem $\left(P_{I g}\right)$ and Problem $\left(P_{I g}^{d}\right)$, we have the following theorem.

Theorem 1 Suppose that $\left(\boldsymbol{\varsigma}^{*}, \boldsymbol{\lambda}^{*}\right)$ is a KKT point of Problem $\left(P_{I g}^{d}\right)$. If $G\left(\boldsymbol{\varsigma}^{*}, \boldsymbol{\lambda}^{*}\right) \succ 0$, then $\boldsymbol{h}^{*}=\frac{1}{2} G^{-1}\left(\boldsymbol{\varsigma}^{*}, \boldsymbol{\lambda}^{*}\right) \boldsymbol{g}$ is the global minimizer of Problem $\left(P_{I g}\right)$. Furthermore, $\tilde{P}_{\boldsymbol{g}}\left(\boldsymbol{h}^{*}\right)=P_{I g}^{d}\left(\boldsymbol{\varsigma}^{*}, \boldsymbol{\lambda}^{*}\right)$.

Proof. 1). We first show that $\boldsymbol{h}^{*}$ is a feasible solution of Problem $\left(P_{I g}\right)$. Furthermore, $\tilde{P}_{\boldsymbol{g}}\left(\boldsymbol{h}^{*}\right)=P_{I g}^{d}\left(\boldsymbol{\varsigma}^{*}, \boldsymbol{\lambda}^{*}\right)$. 
Since $\left(\boldsymbol{\varsigma}^{*}, \boldsymbol{\lambda}^{*}\right)$ is a KKT point of Problem $\left(P_{I g}^{d}\right)$, there exists a $\boldsymbol{\sigma}^{*}$ such that

$$
\left\{\begin{array}{l}
\nabla_{\boldsymbol{\varsigma}} \tilde{L}\left(\boldsymbol{\varsigma}^{*}, \boldsymbol{\lambda}^{*}, \boldsymbol{\sigma}^{*}\right)=0, \nabla_{\boldsymbol{\lambda}} \tilde{L}\left(\boldsymbol{\varsigma}^{*}, \boldsymbol{\lambda}^{*}, \boldsymbol{\sigma}^{*}\right)=0 \\
\left(\boldsymbol{\sigma}^{*}\right)^{T} \boldsymbol{\lambda}^{*}=0 \\
\boldsymbol{\sigma}^{*} \geq 0, \boldsymbol{\lambda}^{*} \geq 0
\end{array}\right.
$$

By direct computation, we have

$$
\nabla_{\boldsymbol{\varsigma}} \tilde{L}\left(\boldsymbol{\varsigma}^{*}, \boldsymbol{\lambda}^{*}, \boldsymbol{\sigma}^{*}\right)=-\frac{1}{2} B^{-1} \boldsymbol{\varsigma}^{*}-\mathbf{1}_{\boldsymbol{M}^{+}}\left[\begin{array}{c}
\frac{1}{4} \boldsymbol{g}^{T} G^{-1}\left(\boldsymbol{\varsigma}^{*}, \boldsymbol{\lambda}^{*}\right) \Psi\left(\omega_{1}^{p}\right) G^{-1}\left(\boldsymbol{\varsigma}^{*}, \boldsymbol{\lambda}^{*}\right) \boldsymbol{g} \\
\ldots \\
\frac{1}{4} \boldsymbol{g}^{T} G^{-1}\left(\boldsymbol{\varsigma}^{*}, \boldsymbol{\lambda}^{*}\right) \Psi\left(\omega_{M}^{p}\right) G^{-1}\left(\boldsymbol{\varsigma}^{*}, \boldsymbol{\lambda}^{*}\right) \boldsymbol{g}
\end{array}\right]
$$

and

$$
\nabla_{\boldsymbol{\lambda}} \tilde{L}\left(\boldsymbol{\varsigma}^{*}, \boldsymbol{\lambda}^{*}, \boldsymbol{\sigma}^{*}\right)=-\varepsilon \mathbf{1}_{\boldsymbol{N}}+\boldsymbol{\sigma}^{*}+\left[\begin{array}{c}
\frac{1}{4} \boldsymbol{g}^{T} G^{-1}\left(\boldsymbol{\varsigma}^{*}, \boldsymbol{\lambda}^{*}\right) \Psi\left(\omega_{1}^{s}\right) G^{-1}\left(\boldsymbol{\varsigma}^{*}, \boldsymbol{\lambda}^{*}\right) \boldsymbol{g} \\
\ldots \\
\frac{1}{4} \boldsymbol{g}^{T} G^{-1}\left(\boldsymbol{\varsigma}^{*}, \boldsymbol{\lambda}^{*}\right) \Psi\left(\omega_{N}^{s}\right) G^{-1}\left(\boldsymbol{\varsigma}^{*}, \boldsymbol{\lambda}^{*}\right) \boldsymbol{g}
\end{array}\right] .
$$

where $\mathbf{1}_{\boldsymbol{M}} \in \mathbb{R}^{M}=[1,1, \cdots, 1]^{T}$. Substituting $\boldsymbol{h}^{*}=\frac{1}{2} G^{-1}\left(\boldsymbol{\varsigma}^{*}, \boldsymbol{\lambda}^{*}\right) \boldsymbol{g}$ into $(26)$ and noting that $\nabla_{\boldsymbol{\lambda}} \tilde{L}\left(\boldsymbol{\varsigma}^{*}, \boldsymbol{\lambda}^{*}, \boldsymbol{\sigma}^{*}\right)=0$ and $\boldsymbol{\sigma}^{*} \geq 0$, we obtain

$$
\left(\boldsymbol{h}^{*}\right)^{T} \Psi\left(\omega_{n}^{s}\right)\left(\boldsymbol{h}^{*}\right)-\varepsilon=-\sigma_{n}^{*} \leq 0 .
$$

Thus, $\boldsymbol{h}^{*}=\frac{1}{2} G^{-1}\left(\boldsymbol{\varsigma}^{*}, \boldsymbol{\lambda}^{*}\right) \boldsymbol{g}$ is a feasible point of Problem $\left(P_{I g}\right)$. Furthermore,

$$
\lambda_{n}^{*}\left(\left(\boldsymbol{h}^{*}\right)^{T} \Psi\left(\omega_{n}^{s}\right)\left(\boldsymbol{h}^{*}\right)-\varepsilon\right)=-\lambda_{n}^{*} \sigma_{n}^{*}=0
$$


Let $\boldsymbol{\xi}^{*}=\left[\left(\boldsymbol{h}^{*}\right)^{T} \Psi\left(\omega_{1}^{p}\right) \boldsymbol{h}^{*}-1, \cdots,\left(\boldsymbol{h}^{*}\right)^{T} \Psi\left(\omega_{M}^{p}\right) \boldsymbol{h}^{*}-1\right]^{T}$. Then, it follows from (25) and $\nabla_{\boldsymbol{\varsigma}} \tilde{L}\left(\boldsymbol{\varsigma}^{*}, \boldsymbol{\lambda}^{*}, \boldsymbol{\sigma}^{*}\right)=0$ that

$$
\boldsymbol{\varsigma}^{*}=2 B \boldsymbol{\xi}^{*}=\nabla V\left(\boldsymbol{\xi}^{*}\right) .
$$

Thus, $\boldsymbol{\varsigma}^{*}$ and $\boldsymbol{\xi}^{*}$ is a conjugate duality pair and the following equality holds

$$
\left(\boldsymbol{\varsigma}^{*}\right)^{T} \boldsymbol{\xi}^{*}=V\left(\boldsymbol{\xi}^{*}\right)+V^{*}\left(\boldsymbol{\varsigma}^{*}\right) .
$$

Therefore,

$$
\begin{aligned}
& \tilde{P}_{\boldsymbol{g}}\left(\boldsymbol{h}^{*}\right)=\alpha\left(\boldsymbol{h}^{*}\right)^{T} D \boldsymbol{h}^{*}+V\left(\boldsymbol{\xi}^{*}\right)-\boldsymbol{g}^{T} \boldsymbol{h}^{*} \\
& =\bar{L}\left(\boldsymbol{h}^{*}, \boldsymbol{\xi}^{*}, \boldsymbol{\lambda}^{*}\right) \quad(\text { by }(27)) \\
& =\Xi\left(\boldsymbol{h}^{*}, \boldsymbol{\varsigma}^{*}, \boldsymbol{\lambda}^{*}\right) \quad(\text { by }(28)) .
\end{aligned}
$$

It is easy to verify that $\Xi\left(\boldsymbol{h}^{*}, \boldsymbol{\varsigma}^{*}, \boldsymbol{\lambda}^{*}\right)=P_{I g}^{d}\left(\boldsymbol{\varsigma}^{*}, \boldsymbol{\lambda}^{*}\right)$ since $\boldsymbol{h}^{*}=\frac{1}{2} G^{-1}\left(\boldsymbol{\varsigma}^{*}, \boldsymbol{\lambda}^{*}\right) \boldsymbol{g}$. Thus,

$$
\tilde{P}_{g}\left(\boldsymbol{h}^{*}\right)=\Xi\left(\boldsymbol{h}^{*}, \varsigma^{*}, \boldsymbol{\lambda}^{*}\right)=P_{I g}^{d}\left(\boldsymbol{\varsigma}^{*}, \boldsymbol{\lambda}^{*}\right) .
$$

$2)$. Now we show that $\boldsymbol{h}^{*}=\frac{1}{2} G^{-1}\left(\boldsymbol{\varsigma}^{*}, \boldsymbol{\lambda}^{*}\right) \boldsymbol{g}$ is the global minimizer of Problem $\left(P_{I g}\right)$. Since $\boldsymbol{h}^{*}$ is a feasible solution, we only need to show that for any $\boldsymbol{h}$, we have

$$
\tilde{P}_{g}(\boldsymbol{h}) \geq \tilde{P}_{g}\left(\boldsymbol{h}^{*}\right), \forall \boldsymbol{h} \text { satisfying }(14)
$$


In fact, for any $\boldsymbol{h}$ satisfying (14), we have

$$
\begin{aligned}
\tilde{P}_{g}(\boldsymbol{h}) \geq & \alpha \boldsymbol{h}^{T} D \boldsymbol{h}+(1-\alpha) \sum_{m=1}^{M} \nu_{m}\left(\boldsymbol{h}^{T} \Psi\left(\omega_{m}^{p}\right) \boldsymbol{h}-1\right)^{2} \\
& -\boldsymbol{g}^{T} \boldsymbol{h}+\sum_{n=1}^{N} \lambda_{n}^{*}\left(\boldsymbol{h}^{T} \Psi\left(\omega_{n}^{s}\right) \boldsymbol{h}-\varepsilon\right) \quad(\boldsymbol{h} \text { satisfying }(14)) \\
\geq & \sum_{m=1}^{M} \varsigma_{m}^{*}\left(\boldsymbol{h}^{T} \Psi\left(\omega_{m}^{p}\right) \boldsymbol{h}-1\right)-\frac{1}{4}\left(\boldsymbol{\varsigma}^{*}\right)^{T} B^{-1} \boldsymbol{\varsigma}^{*} \\
& +\sum_{n=1}^{N} \lambda_{n}^{*}\left(\boldsymbol{h}^{T} \Psi\left(\omega_{n}^{s}\right) \boldsymbol{h}-\varepsilon\right)+\alpha \boldsymbol{h}^{T} D \boldsymbol{h}-\boldsymbol{g}^{T} \boldsymbol{h} \\
& \left(\text { by the inequality } V(\boldsymbol{\xi})+V\left(\boldsymbol{\varsigma}^{*}\right) \geq\left(\boldsymbol{\varsigma}^{*}\right)^{T} \boldsymbol{\xi}\right) \\
= & \Xi\left(\boldsymbol{h}, \boldsymbol{\varsigma}^{*}, \boldsymbol{\lambda}^{*}\right) .
\end{aligned}
$$

Note that $\Xi(\boldsymbol{h}, \boldsymbol{\varsigma}, \boldsymbol{\lambda})$ is a quadratic function with respect to $\boldsymbol{h}$ and that

$$
\nabla_{\boldsymbol{h}} \Xi\left(\boldsymbol{h}^{*}, \boldsymbol{\varsigma}^{*}, \boldsymbol{\lambda}^{*}\right)=2 G\left(\boldsymbol{\varsigma}^{*}, \boldsymbol{\lambda}^{*}\right) \boldsymbol{h}^{*}-\boldsymbol{g}=0 .
$$

By these together with (29) and (30), it yields

$$
\begin{aligned}
\tilde{P}_{g}(\boldsymbol{h})-\tilde{P}_{g}\left(\boldsymbol{h}^{*}\right) \geq & \Xi\left(\boldsymbol{h}, \boldsymbol{\varsigma}^{*}, \boldsymbol{\lambda}^{*}\right)-\Xi\left(\boldsymbol{h}^{*}, \boldsymbol{\varsigma}^{*}, \boldsymbol{\lambda}^{*}\right) \\
= & \left(\nabla_{\boldsymbol{h}} \Xi\left(\boldsymbol{h}^{*}, \boldsymbol{\varsigma}^{*}, \boldsymbol{\lambda}^{*}\right)\right)^{T}\left(\boldsymbol{h}-\boldsymbol{h}^{*}\right) \\
& +\frac{1}{2}\left(\boldsymbol{h}-\boldsymbol{h}^{*}\right)^{T} \nabla_{\boldsymbol{h} \boldsymbol{h}}^{2} \Xi\left(\boldsymbol{h}^{*}, \boldsymbol{\varsigma}^{*}, \boldsymbol{\lambda}^{*}\right)\left(\boldsymbol{h}-\boldsymbol{h}^{*}\right) \\
= & \left(\boldsymbol{h}-\boldsymbol{h}^{*}\right)^{T} G\left(\boldsymbol{\varsigma}^{*}, \boldsymbol{\lambda}^{*}\right)\left(\boldsymbol{h}-\boldsymbol{h}^{*}\right) \\
\geq & 0, \forall \boldsymbol{h} \text { satisfying }(14) .
\end{aligned}
$$

We complete the proof. 
Theorem 1 shows that the global solution of Problem $\left(P_{I g}\right)$ may be obtained through solving its canonical dual problem $\left(P_{I g}^{d}\right)$. Since Problem $\left(P_{I g}\right)$ is a small perturbation of Problem $\left(P_{I}\right)$, we can use a gradient-based optimization technique to obtain an improved solution. After obtaining the solution of Problem $\left(P_{I}\right)$, we check whether this solution satisfies the functional inequality constraint of Problem (P). If it does, this solution is further refined by a gradient-based optimization method and the corresponding solution obtained is viewed as an approximate solution of Problem $(\mathrm{P})$. Otherwise, we increase the discretization number and repeat the above process.

To proceed further, we need Schur complement lemma [20].

\section{Lemma 2 Let}

$$
A=\left(\begin{array}{cc}
B_{1} & B_{2}^{T} \\
B_{2} & B_{3}
\end{array}\right),
$$

where $B_{1}$ and $B_{3}$ are square matrices and $B_{2}$ is a matrix with proper dimension. If $B_{1} \succ 0$, then $A$ is positive semi-definite if and only if the matrix $B_{3}-B_{2} B_{1}^{-1} B_{2}^{T}$ is positive semi-definite.

To solve Problem $\left(P_{I g}^{d}\right)$, we note that the matrix inverse is involved in the objective function (20). By exploiting its structure, the main theorem of the paper is obtained by combining Theorem 1 and Lemma 2 as follows.

Theorem 3 Let $\left(t_{1}^{*}, t_{2}^{*}, \boldsymbol{\varsigma}^{*}, \boldsymbol{\lambda}^{*}\right)$ be the solution of the following semi-definite 
programming problem which is referred to as Problem (RSDP)

$$
\begin{aligned}
\min & \frac{1}{4} t_{1}+\frac{1}{4} t_{2}+\sum_{m=1}^{M} \varsigma_{m}+\varepsilon \sum_{n=1}^{N} \lambda_{n} \\
\text { subject to } & {\left[\begin{array}{cc}
G(\boldsymbol{\varsigma}, \boldsymbol{\lambda}) & \boldsymbol{g} \\
\boldsymbol{g}^{T} & t_{1}
\end{array}\right] \succeq 0, } \\
& {\left[\begin{array}{ll}
B & \boldsymbol{\varsigma} \\
\boldsymbol{\varsigma}^{T} & t_{2}
\end{array}\right] \succeq 0, } \\
& \boldsymbol{\lambda} \geq 0 .
\end{aligned}
$$

If $G\left(\boldsymbol{\varsigma}^{*}, \boldsymbol{\lambda}^{*}\right) \succ 0$, then $\boldsymbol{h}^{*}=\frac{1}{2} G^{-1}\left(\boldsymbol{\varsigma}^{*}, \boldsymbol{\lambda}^{*}\right) \boldsymbol{g}$ is the global minimizer of Problem $\left(P_{I g}\right)$.

Proof. The validity of the results follows readily from Lemma 2 and Theorem 1. We complete the proof.

Problem (RSDP) is a linear SDP problem which can be easily solved by existing SDP software packages, for example, SeDuMi, [21]. Theorem 3 shows that solving Problem (RSDP) produces a global minimizer of Problem $\left(P_{I g}^{d}\right)$ if $G\left(\boldsymbol{\varsigma}^{*}, \boldsymbol{\lambda}^{*}\right) \succ 0$. However, if $G\left(\boldsymbol{\varsigma}^{*}, \boldsymbol{\lambda}^{*}\right)$ is singular, there is still no way to dig out a global solution of Problem $\left(P_{I g}\right)$ from the solution of Problem (RSDP). Fortunately, this case is rather rare for our problem. Now we are in a position to present an algorithm for solving Problem $(\mathrm{P})$ as follows.

\section{Algorithm 1}


Step 1. Initialization $K, M$ and $N$.

Step 2. Randomly produce the small perturbation vector $\boldsymbol{g}$.

Step 3. Solve Problem (RSDP) and output solution $\left(t_{1}^{*}, t_{2}^{*}, \boldsymbol{\varsigma}^{*}, \boldsymbol{\lambda}^{*}\right)$. If $G\left(\boldsymbol{\varsigma}^{*}, \boldsymbol{\lambda}^{*}\right) \succ$ 0 , let $\boldsymbol{h}_{i g}^{*}=\frac{1}{2} G^{-1}\left(\boldsymbol{\varsigma}^{*}, \boldsymbol{\lambda}^{*}\right) \boldsymbol{g}$, go to Step 4. Otherwise, go to Step 2.

Step 4. Using $\boldsymbol{h}_{i g}^{*}$ as an initial condition, solve Problem $(P)$ with the functional inequality constraint (10) replaced by the approximate discritized constraints (14) by a gradient-based optimization method. Check whether the functional inequality constraint (10) is satisfied in a dense set of $B_{s}$. If it is, stop and output the solution $\boldsymbol{h}^{*}$. Otherwise, increase $N$ and goto Step 2.

\section{Numerical Experiments}

Let the desired magnitude response be given by

$$
\left|H_{d}(\omega)\right|=\left\{\begin{array}{l}
1, \text { if } \omega \in[0,0.12 \pi], \\
0, \text { if } \omega \in[0.24 \pi, \pi]
\end{array}\right.
$$

and $\alpha$ is chosen from $\left[10^{-8}, 10^{-4}\right]$.

During our simulation, we set $K=32, M=50, N=200$ and $\varepsilon=$ $-35 \mathrm{~dB}$. Algorithm 1 is used to solve the optimization problem (13) and (14) with different values of $\alpha$. The small perturbation vector is generated by $10^{-5} \operatorname{randn}(K+1,1)$. The weights $\nu_{m}$ and the Gaussian quadrature points 
$\omega_{m}^{p}, m=1, \cdots, M$, can be found from [22]. Problem (RSDP) is solved by SeDuMi [21] together with YALMIP [23] and the nonlinear constrained optimization is solved by "fmincon" in the Optimization Toolbox within the Matlab environment.

For comparison, we use the existing convex optimization methods used in $[7,24]$ in conjunction with a minimum phase spectral factorization method [25] to design a low group delay filter under the same criteria. More specifically, taking $\alpha=0$ in (13), replacing $|H(\omega)|^{2}$ by $R(\omega)=r_{0}+\sum_{k=1}^{K} 2 r_{k} \cos (k \omega)=$ $\boldsymbol{r}^{T} \boldsymbol{\psi}(\omega)$ (where $\boldsymbol{r}=\left[r_{0}, \cdots, r_{K}\right]^{T}$ and $\left.\boldsymbol{\psi}(\omega)=[1,2 \cos (\omega), \cdots, 2 \cos ((K \omega))]\right)$ and imposing a linear matrix inequality [24] to ensure the invertibility from $\boldsymbol{r}$ to $\boldsymbol{h}$, a quadratic optimization problem with a linear matrix inequality and linear constraints in terms of $\boldsymbol{r}$ and an auxiliary matrix variable $P_{c}$ is obtained as follows:

$$
\begin{aligned}
& \min _{\boldsymbol{r}, P_{c}} \sum_{m=1}^{M} \nu_{m}\left(\boldsymbol{r}^{T} \boldsymbol{\psi}\left(\omega_{m}^{p}\right)-1\right)^{2} \\
& \text { subject to } \quad \boldsymbol{r}^{T} \boldsymbol{\psi}\left(\omega_{n}^{s}\right) \leq \varepsilon, n=1, \cdots, N . \\
& {\left[\begin{array}{cc}
P_{c}-A_{c}^{T} P_{c} A_{c} & C_{c}^{T}-A_{c}^{T} P_{c} B_{c} \\
C_{c}-B_{c}^{T} P_{c} A_{c} & D_{c}+D_{c}^{T}-B_{c}^{T} P_{c} B_{c}
\end{array}\right] \succeq 0,}
\end{aligned}
$$

where $A_{c}, B_{c}, C_{c}, D_{c}$ are given in (7) in [24]. After finding the solution of the optimization problem defined by (35), the minimum phase spectral factorization method introduced in [25] is used to extract $\boldsymbol{h}$ from $\boldsymbol{r}$. Let the solution obtained be denoted as $\boldsymbol{h}_{c}^{*}$. The computational complexity to obtain 
$\boldsymbol{h}_{\alpha}^{*}$ and $\boldsymbol{h}_{c}^{*}$ are similar as both Problem (RSDP) and Problem (35) involve a linear matrix inequality. However, Problem (35) contains more decision variables. Furthermore, after the solution of Problem (35) is obtained, a minimum phase spectral factorization is required to be used to extract $\boldsymbol{h}_{c}^{*}$ from the solution $\boldsymbol{r}^{*}$.

We first set $\alpha_{1}=10^{-6}$ and use Algorithm 1 to solve Problem $\left(P_{I}\right)$. Let the solution obtained be denoted as $\boldsymbol{h}_{\alpha_{1}}^{*}$. During the solution process, the function "fmincon" just takes two iterations to stop. In fact, the performance of the solution obtained by solving Problem (RSDP) has no visible difference from that refined by "fmincon". Thus, the gradient-based optimization refinement seems not necessary from our computational experience. Nonetheless, it is included in Algorithm 1, as it may be needed in other problems.

The results corresponding to $\boldsymbol{h}_{\alpha_{1}}^{*}$ and $\boldsymbol{h}_{c}^{*}$ are depicted in Fig. 1. From Fig. 1 (b), we see that there is no visible difference between the magnitude response of $\boldsymbol{h}_{\alpha_{1}}^{*}$ and that of $\boldsymbol{h}_{c}^{*}$ in the passband. Fig. 1 (c) shows that both $\boldsymbol{h}_{\alpha_{1}}^{*}$ and $\boldsymbol{h}_{c}^{*}$ are with the same stopband ripples. However, it is observed in Fig. 1 (d) that $\boldsymbol{h}_{\alpha_{1}}^{*}$ achieves a much smaller group delay than $\boldsymbol{h}_{c}^{*}$. Fig. 1 (e) shows that all zeros of $\boldsymbol{h}_{\alpha_{1}}^{*}$ are within the unit circle. Thus, the filter $\boldsymbol{h}_{\alpha_{1}}^{*}$ designed by our method is automatically a minimum phase filter without having to performing a minimum phase extraction step. This is not shared by any existing method. From this example, it is clear that our method can 
achieve a much smaller group delay while keeping the same design criteria.

To investigate the impact of the parameter $\alpha$, we increase $\alpha$ from $\alpha_{1}$ to $\alpha_{2}=10^{-5}$. Let the solution obtained by our method be denoted as $\boldsymbol{h}_{\alpha_{2}}^{*}$. The results corresponding to $\boldsymbol{h}_{\alpha_{2}}^{*}$ and $\boldsymbol{h}_{c}^{*}$ are depicted in Fig. 2. Fig. 2 shows that the group delay of $\boldsymbol{h}_{\alpha_{2}}^{*}$ becomes much smaller at the cost of some increase in its magnitude response in the passband and stopband.

Now we decrease $\alpha$ from $\alpha_{1}$ to $\alpha_{3}=10^{-7}$. Let the solution obtained by our method be denoted as $\boldsymbol{h}_{\alpha_{3}}^{*}$. The results corresponding to $\boldsymbol{h}_{\alpha_{3}}^{*}$ and $\boldsymbol{h}_{c}^{*}$ are depicted in Fig. 3. Fig. 3 shows that both the magnitude response and the group delay of $\boldsymbol{h}_{\alpha_{3}}^{*}$ are close to $\boldsymbol{h}_{c}^{*}$. In theory, $\boldsymbol{h}_{c}^{*}$ can be obtained from $\boldsymbol{h}_{\alpha}^{*}$ by setting $\alpha \rightarrow 0$. All the coefficients of $\boldsymbol{h}_{\alpha_{1}}^{*}, \boldsymbol{h}_{\alpha_{2}}^{*}, \boldsymbol{h}_{\alpha_{3}}^{*}$ and $\boldsymbol{h}_{c}^{*}$ are presented in Table 1.

Clearly, the parameter $\alpha$ plays an important role during our design process. In fact, the role of $\alpha$ is two-fold. On one hand, it is used to control the trade-off between the group delay and the magnitude response. The larger the $\alpha$ is, the smaller the group delay is achieved. Since the term $\sum_{m=1}^{M} \nu_{m}\left(\boldsymbol{h}^{T} \Psi\left(\omega_{m}^{p}\right) \boldsymbol{h}-1\right)^{2}$ is much smaller than the term $\boldsymbol{h}^{T} D \boldsymbol{h}$, we usually take the value of $\alpha$ starting from $10^{-4}$. If the resulting magnitude response does not meet our desired specification, we decrease the value of $\alpha$ by $\alpha=\alpha / 10$ or to a smaller one. On the other hand, $\alpha$ plays the role of regularization during the process of solving Problem (RSDP). If the value of 
$\alpha$ is too small (for example, smaller than $10^{-8}$ ), Problem (RSDP) may not admit a solution such that $G\left(\boldsymbol{\varsigma}^{*}, \boldsymbol{\lambda}^{*}\right) \succ 0$. At the first glance, the adjustment of $\alpha$ appears to increase the complexity of solving the problem. In fact, due to the presence of $\alpha$, we have more freedom to control the trade-off between the magnitude response and the group delay. Furthermore, a proper choice of $\alpha$ can lead to a smaller group delay while keeping all the design criteria the same (see Fig. 1).

\section{Conclusion}

In this paper, the design of a low group delay FIR filter is studied. Unlike the conventional minimum phase method, we formulate it as a non-convex optimization problem directly. Our main contribution is that a novel numerical scheme is developed to solve this formulated non-convex optimization problem globally. Numerical experiments show that our design method can produce a filter with smaller group delay than those obtained by the existing convex optimization method used in conjunction with a minimum phase extraction method under the same design criteria.

\section{References}

[1] G. Apaydin, Realization of reduced-delay finite impulse response filters for audio applications, Digital Signal Process., Vol. 20, (2010) 620-629. 
[2] G. Calvagno, G. M. Cortelazzo and G. A. Mian, A technique for multiple criterion approximation of FIR filters in magnitude and group delay, IEEE Trans. Signal Process., Vol 43 (1995) 393-400.

[3] T. Stathaki and I. Fotinopoulos, Equiripple minimum phase FIR filter design from linear phase systems using root moments, IEEE Trans. Circuits Syst. II, Analog Digit. Signal Process., Vol. 48, (2001) 580-587.

[4] X.P. Lai, Optimal design of nonlinear-phase FIR filters with prescribed phase error, IEEE Trans. Signal Process., Vol. 57 (2009) 3399-3410.

[5] Z.P. Lin and Y.Z. Liu, Design of complex FIR filters with reduced group delay error using semidefinite programming, IEEE Signal Process. Lett., Vol 13 (2006) 529-532.

[6] Z.Q., Luo, Applications of convex optimization in signal processing and digital communication, Math. Program., Vol 97 (2003) 177-207.

[7] I. M.S. Panahi and K. Venkat, A new class of invertible FIR filters for spectral shaping, Signal Processing, Vol. 89 (2009) 2128-2142.

[8] S.C. Pei and S.T. Lu, Design of minimum-phase FIR filters by differential cepstrum, IEEE Trans. Circuits Syst., Vol. 33 (1986) 570-576.

[9] H. J. Orchard and A. N. Willson, On the computation of a minimum 
phase spectral factor, IEEE Trans. Circuits Syst. I, Fundam. Theory Appl., Vol. 50 (2003) 365-375.

[10] O. Herrmann and W. Schuessler, Design of nonrecursive digital filters with minimum phase, Electron. Lett., Vol. 6, (1970) 329-330.

[11] S.C. Pei and H.S. Lin, Minimum-phase FIR filter design using real cepstrum, IEEE Trans. Circuits Syst. II, Vol. 53 (2006) 1113-1117.

[12] C. Rusu and J. Astola, Minimum-phase parts of zero-phase sequences, Signal Processing, Vol. 89 (2009) 1032-1037.

[13] Y.C. Shih and H.C. Wu, Theoretical analysis on the finite-support approximation for the mixing-phase FIR systems, Signal Processing Vol. 89 (2009) 2128-2142.

[14] I.M.S. Panahi and K. Venkat, A new class of invertible FIR filters for spectral shaping, Signal Processing 89 (2009) 1271-1287.

[15] D.Y. Gao, Duality Principles in Nonconvex Systems: Theory, Methods and Applications. Kluwer Academic, Dordrecht (2000).

[16] D. Gao and C.Z. Wu, On the triality theory for a quartic polynomial optimization problem, J. Ind. Manag. Optim., Vol. 8, (2012) 229-242.

[17] T. Kurbiel, H.G. Gockler and D. Alfsmann, A novel approach to the design of oversampling low-delay complex-modulated filter bank pairs, 
EURASP Journal on Advances in Signal Processing, Vol. 2009 (2009) $1-13$.

[18] N. Ruan, D.Y. Gao, Y. Jiao, Canonical dual least square method for solving general nonlinear systems of quadratic equations, Comput. Optim. Appl., Vol. 47 (2010) 335-347.

[19] R.T. Rockafellar, Convex Analysis, Princeton University Press, Princeton, NJ., 1970.

[20] S. Boyd, L.E. Ghaoui, E. Feron and V. Balarkrishnan, Linear matrix inequalities in system and control theory, Philadelphia, PA: SIAM, Vol. 15, Studies in Applied Mathematics, 1994.

[21] J. F. Sturm, Using SeDuMi 1.02, a Matlab toolbox for optimization over symmetric cones, Optimiz. Methods Softw., Vol 11-12 (1999) 625-653.

[22] http://processingjs.nihongoresources.com/bezierinfo/legendre-gaussvalues.php

[23] J. Löberg, YALMIP : A toolbox for modeling and optimization in Matlab, Proc. Int. Symp. CACSD, Taipei, Taiwan, Sep. 2004, pp. 284-89.

[24] S.-P. Wu, S. Boyd, and L. Vandenberghe, FIR filter design via semidefinite programming and spectral factorization, in Proc. IEEE Conf. Decision Contr., 1996, pp. 271-276. 
[25] A. Papoulis. Signal Analysis. McGraw-Hill, New York, 1977. 
Table 1: The coefficients of $\boldsymbol{h}_{\alpha_{1}}^{*}, \boldsymbol{h}_{\alpha_{2}}^{*}, \boldsymbol{h}_{\alpha_{3}}^{*}$, and $\boldsymbol{h}_{c}^{*}$

\begin{tabular}{|c|c|c|c|}
\hline $\boldsymbol{h}_{\alpha_{1}}^{*}$ & $\boldsymbol{h}_{\alpha_{2}}^{*}$ & $\boldsymbol{h}_{\alpha_{3}}^{*}$ & $\boldsymbol{h}_{c}^{*}$ \\
\hline 0.0285116206006799 & 0.0387740383889875 & 0.0262107023409039 & 0.0252472337305588 \\
\hline 0.0509866524174902 & 0.0614443952270321 & 0.0486333750106783 & 0.0473450207730468 \\
\hline 0.0856088083718892 & 0.0978828806389367 & 0.0822097183380445 & 0.0810379492029237 \\
\hline 0.123034390901257 & 0.134719509319526 & 0.119931194418926 & 0.118315645903861 \\
\hline 0.156084821647626 & 0.164035324986959 & 0.153337081728234 & 0.152127711650623 \\
\hline 0.17639025618915 & 0.178537316915211 & 0.175252349657358 & 0.174612661066523 \\
\hline 0.178278138164555 & 0.173044184628575 & 0.178831620770272 & 0.179080069523033 \\
\hline 0.158868245465607 & 0.146945417351412 & 0.16138455335902 & 0.162414973421189 \\
\hline 0.120358748692553 & 0.104316502480046 & 0.124389155519067 & 0.12617170171523 \\
\hline 0.0698444119645312 & 0.0532464367514738 & 0.0745058664743655 & 0.0766929272231663 \\
\hline 0.0171576799182006 & 0.00358296855479145 & 0.0216618169750614 & 0.0236538095680745 \\
\hline-0.0273048888932891 & -0.03523190002442 & -0.0240601445359459 & -0.0224972156907095 \\
\hline-0.055408731398201 & -0.0569798418610146 & -0.0540575198345802 & -0.0532595049087454 \\
\hline-0.063955965089311 & -0.0597728525689495 & -0.0642092858615103 & -0.0643882215963533 \\
\hline-0.0542237462602092 & -0.0468743219339164 & -0.0560442066213009 & -0.0567683880468747 \\
\hline-0.0322817257959678 & -0.024592722813039 & -0.0348589697777236 & -0.0358287251589483 \\
\hline-0.00627605177132923 & -0.000912831822104257 & -0.00842407441488813 & -0.00965476219362919 \\
\hline 0.0158289990856661 & 0.0177061601908936 & 0.014330704665103 & 0.0135807646076558 \\
\hline 0.0286195549008114 & 0.0269920614387458 & 0.0280939492720026 & 0.0279162706674796 \\
\hline 0.0302151504788049 & 0.0265448718017831 & 0.0309081516924011 & 0.0310432735978916 \\
\hline 0.0227853602802973 & 0.0185592874179371 & 0.0239044204015682 & 0.0244040175111291 \\
\hline 0.0102400250794694 & 0.00737664537854957 & 0.011449384382796 & 0.0120974536216775 \\
\hline-0.0022899340896227 & -0.00297170649287577 & -0.00127364695662734 & -0.0008551248303386 \\
\hline-0.0109135754408663 & -0.00952784396092289 & -0.0105402761284925 & -0.0103020083473471 \\
\hline-0.0139437136272584 & -0.0114233791995216 & -0.0141124838925119 & -0.0141101882320104 \\
\hline-0.0116738095909395 & -0.00928896815384196 & -0.0121975868915368 & -0.0124765774288458 \\
\hline-0.00633401566033477 & -0.00495774606814332 & -0.00708446025529165 & -0.00735359258744947 \\
\hline-0.000742918188205383 & -0.000457217685890348 & -0.00113687290785976 & -0.00137683502708685 \\
\hline 0.00366739459678194 & 0.00252396781666946 & 0.00329482070235785 & 0.00326212338320078 \\
\hline 0.00540636197896201 & 0.00360183317036392 & 0.00574885041723123 & 0.00551582780234008 \\
\hline 0.00510626880733615 & 0.00295381264416561 & 0.00534983833018261 & 0.00571431177419455 \\
\hline 0.00392200136977837 & 0.00152181737685866 & 0.00482044693188607 & 0.00515350178800323 \\
\hline-0.00524060872051433 & -0.000768122189310436 & -0.00592857341991079 & -0.00619364401091036 \\
\hline
\end{tabular}




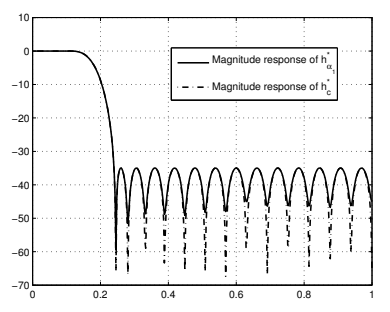

(a)

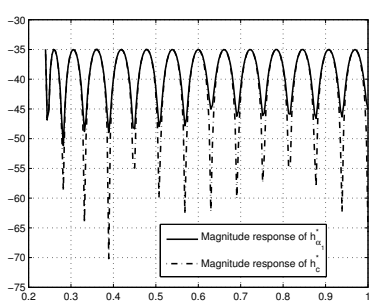

(c)

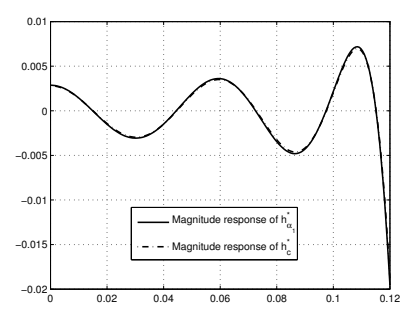

(b)

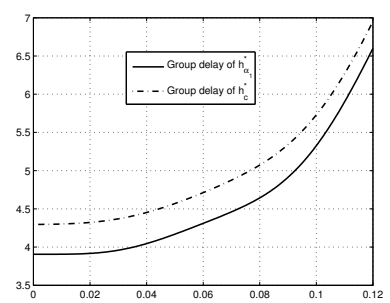

(d)

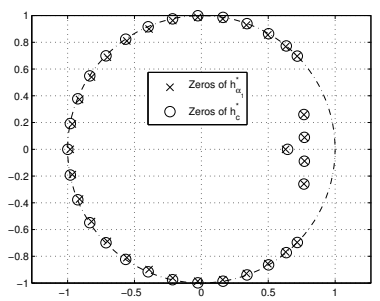

(e)

Figure 1: Magnitude responses and group delays of $\boldsymbol{h}_{\alpha_{1}}^{*}$ and $\boldsymbol{h}_{c}^{*}$ : (a) The magnitude response (in $\mathrm{dB}$ ), (b) Zoom-in of the magnitude response in the passband (in dB), (c) Zoom-in of the magnitude response in the stopband (in $\mathrm{dB}$ ), (d) The group delay in the passband, (e) Zeros of FIR filters of $\boldsymbol{h}_{\alpha_{1}}^{*}$ and $\boldsymbol{h}_{c}^{*}$ 


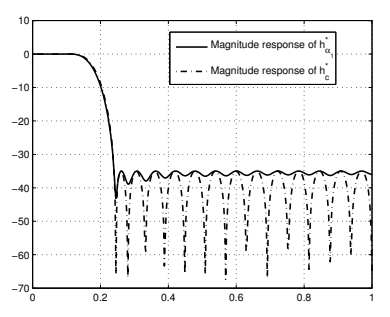

(a)

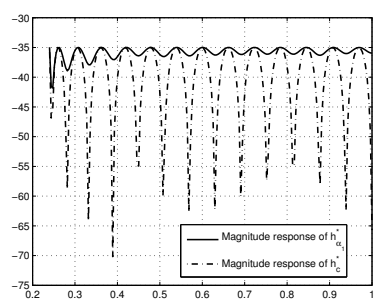

(c)

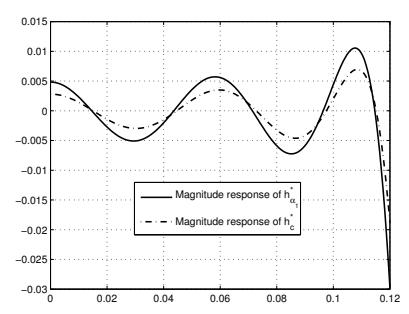

(b)

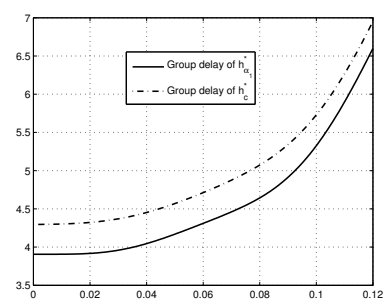

(d)

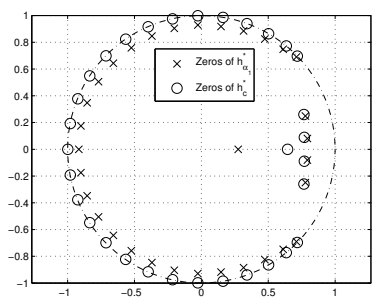

(e)

Figure 2: Magnitude responses and group delays of $\boldsymbol{h}_{\alpha_{2}}^{*}$ and $\boldsymbol{h}_{c}^{*}$ : (a) The magnitude response (in $\mathrm{dB}$ ), (b) Zoom-in of the magnitude response in the passband (in dB), (c) Zoom-in of the magnitude response in the stopband (in $\mathrm{dB}$ ), (d) The group delay in the passband, (e) Zeros of FIR filters of $\boldsymbol{h}_{\alpha_{2}}^{*}$ and $\boldsymbol{h}_{c}^{*}$ 


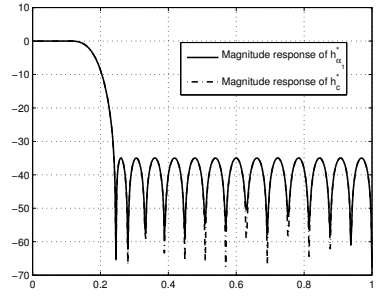

(a)

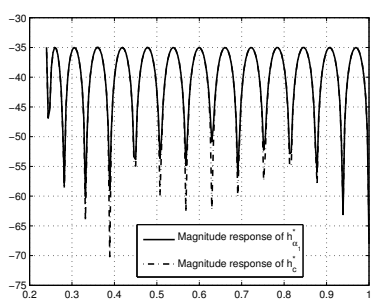

(c)

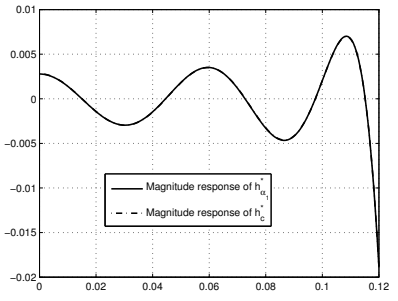

(b)

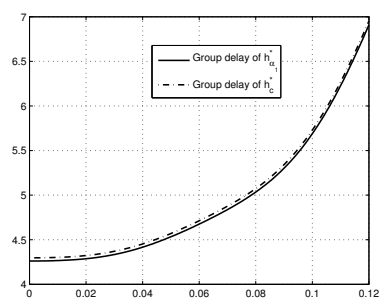

(d)

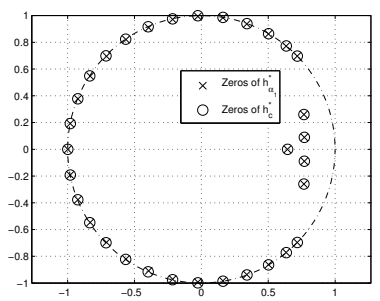

(e)

Figure 3: Magnitude responses and group delays of $\boldsymbol{h}_{\alpha_{3}}^{*}$ and $\boldsymbol{h}_{c}^{*}$ : (a) The magnitude response (in $\mathrm{dB}$ ), (b) Zoom-in of the magnitude response in the passband (in dB), (c) Zoom-in of the magnitude response in the stopband (in $\mathrm{dB}$ ), (d) The group delay in the passband, (e) Zeros of FIR filters of $\boldsymbol{h}_{\alpha_{3}}^{*}$ and $\boldsymbol{h}_{c}^{*}$ 